\title{
Petrogenesis of Quaternary Shoshonitic Volcanism in NE Iran (Ardabil): Implication for Postcollisional Magmatism
}

\author{
Habib Shahbazi Shiran \\ Department of Archaeology, University of Mohagheghe Ardabili, Ardabil, Iran \\ Correspondence should be addressed to Habib Shahbazi Shiran; shahbazihabib@yahoo.com
}

Received 3 April 2013; Revised 27 August 2013; Accepted 9 October 2013

Academic Editor: Ryszard Kryza

Copyright ( 2013 Habib Shahbazi Shiran. This is an open access article distributed under the Creative Commons Attribution License, which permits unrestricted use, distribution, and reproduction in any medium, provided the original work is properly cited.

\begin{abstract}
Trachyandesites, trachytes, andesites, and pyrocalstic rocks, with shoshonitic signature, are the main Quaternary volcanic rocks in the Sabalan region (Ardabil). Plagiocalse, K-feldspar, biotite associated with clinopyroxene, and glass are the main constituents of these lavas. Plagioclases are andesine to labradorite while clinopyroxenes have augitic composition. The Sabalan volcanic rocks show enrichment in LREEs (relative to HREEs) and are characterized by enrichment in LILEs and depletion in HFSEs. Petrological observations, along with rare earth and trace elements geochemistry, suggest shoshonitic signature for Sabalan lavas. This signature highlights derivation from a subduction-related source. The Sabalan volcanic rocks are isotopically characterized by derivation from an enriched mantle source with a tendency to plot in the fields defined by island-arc basalts (IAB) and OIBs (in $\varepsilon$ Nd versus ${ }^{87} \mathrm{Sr} /{ }^{86} \mathrm{Sr}$ diagram). The geochemical and isotopic characteristics of the Sabalan lavas suggest that their magma has been issued via low degree partial melting of a subduction-metasomatized continental lithospheric mantle. The formation of these lavas is related to slab steepening and breakoff in a postcollisional regime.
\end{abstract}

\section{Introduction}

Cenozoic magmatism is well known from the peri-Arabic region, north of the Bitlis-Zagros Suture Zone (Figure 1), and ranges in age from Eocene to Plio-Quaternary. The timing of the eruptions and pooling the plutons mostly coincides with and postdates a series of continental collisional events in the northern Bitlis-Zagros Suture Zone region $[1,2]$. The Eocene magmatism in the peri-Arabic region (both in the Urumieh-Dokhtar magmatic belt and south of the Anatolian block) is calc-alkalic to shoshonitic, which resulted from subduction of the Neotethyan Ocean beneath the central Iranian and Anatolian blocks. The Plio-Quaternary (and Quaternary) alkaline-ultrapotassic magmatism occupies most of the Turkish-Iranian high plateau (NW Iran and NE Turkey) and is mostly characterized by within-plate and/or subductionrelated geochemical characteristics [3-5]. The geochemical variations of late Cenozoic lavas indicate a progression from calc-alkaline to alkaline compositions with time [2].

The Turkish-Iranian high plateau is bounded on the north by the Eastern Pontide arc and Lesser Caucasus magmatic belt and to the south by continental blocks including Bitlis, Puturg, Sanandaj, and Sirjan blocks. PlioQuaternary and/or Quaternary volcanic cones and flows are scattered in this plateau (such as Mt. Ararat, Sahand, Sabalan, Nemrut, and others) and most of them indicate postcollisional shoshonitic-alkaline affinities [7-10]. The Sabalan volcano (Ardabil) is among the youngest volcanic calderas, in eastern border of the Turkish-Iranian Plateau, consisting of Miocene trachyandesites and Plio-Quaternary trachy-andesite, pumiceous andesites, and dacites with ultrapotassic-shoshonitic signatures. The aim of this study is to recognize the major, trace, $\mathrm{REE}$ and $\mathrm{Sr}-\mathrm{Nd}$ isotopes characteristics of the Sabalan Quaternary lavas and to present a consistent tectonomagmatic model for the formation and evolution of the Sabalan volcano.

\section{Regional Geology}

The southwestern Sabalan region is characterized by Miocene magmatism with occurrence of calc-alkaline and high K calcalkaline to shoshonitic lavas [11]. Moreover, late Miocene 


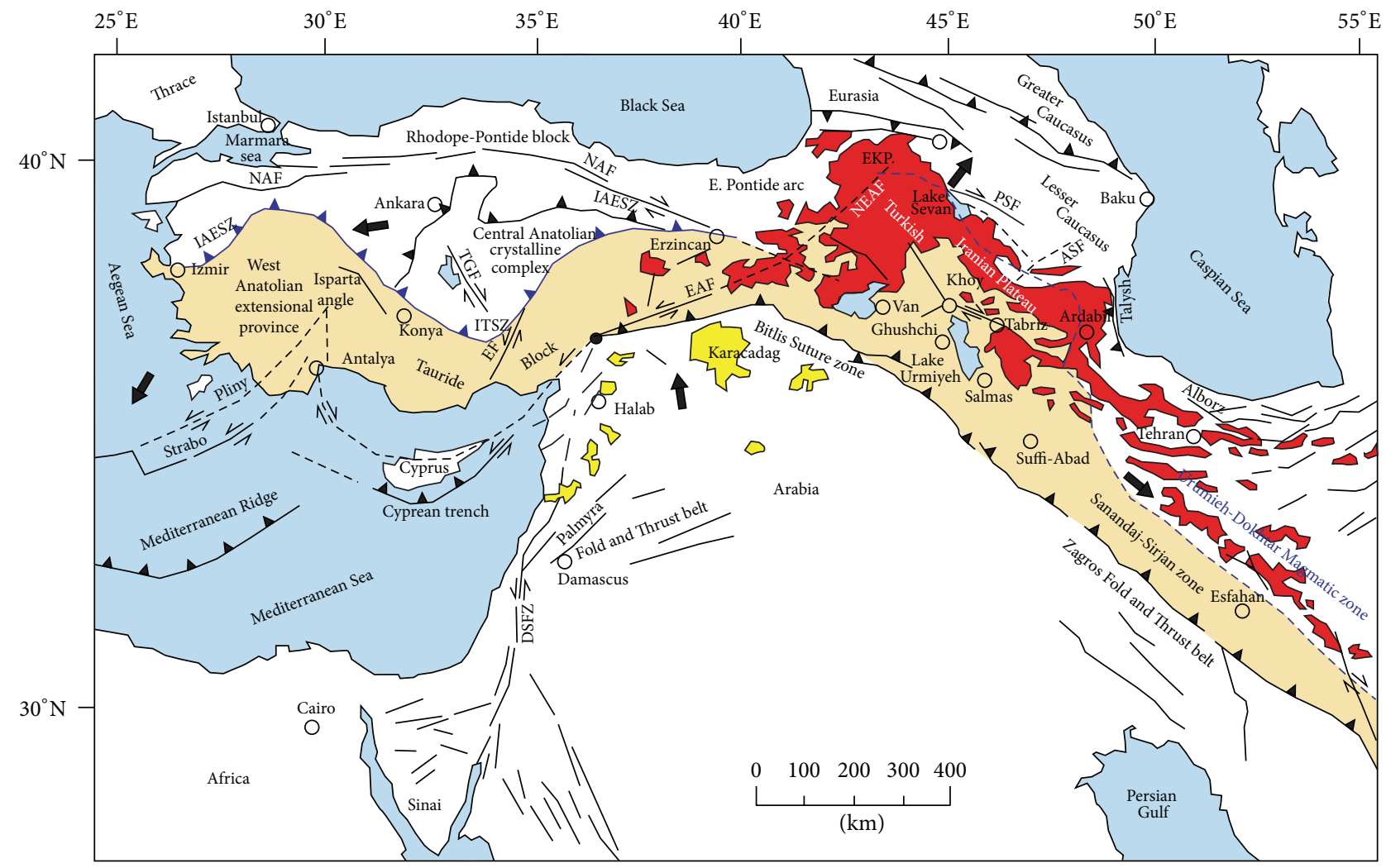
Postcollisional volcanic rocks in
eastern Anatolia, Iran and Caucasus
Rigid blocks with Afro-Arabian
eastern Anatolia, Iran and Caucas
affinity
rocks in Afro-Arabia
Plate convergence vectors
(with respect to fixed Eurasia)

FIGURE 1: Simplified tectonic map of the eastern Mediterranean-Persian Gulf region showing the active plate boundaries and post-collisional volcanic rocks in the Peri-Arabian region modified after [6]. Abbreviation: PSF = Pampak-Sevan Fault; EKP = Erzurum-Kars Plateau; EAF $=$ East Anatolian Fault; IAESZ = Izmir-Ankara-Erzincan Suture Zone; EF = Ecemis Fault; NAF = North Anatolian Fault; DSFZ = Dead Sea Fault Zone.

sodic alkaline lavas are common in south of the Sabalan region [12]. Eocene and Oligo-Miocene lavas are also distributed in NW regions of Ardabil, near Meshkin ShahrAhar volcanic belt. The Eocene lavas are mostly andesites, trachyandesites, trachybasalts and analcime-bearing tephrites with shoshonitic to alkaline geochemical signatures. Volcanic breccias, tuffs, and interbedded lavas, with columnar joints are common in the N-NE of the Sabalan region. Andesitic to analcime-bearing tephritic dikes crosscut the pyroclastic sequence, mainly at NE of Meshkin Shahr. Eocene granitic, monzonitic, and monzogabbroic intrusions are common in the Ahar-Meshkin Shahr magmatic belt. These plutonic rocks have shoshonitic geochemical affinity [13].

The Sabalan volcano consists of both late Miocene (old) and Quaternary (young) trachyandesitic and trachytic to dacitic lavas with huge bodies of ignimbrites-density pyroclastic flows [14]. The Sabalan magmatic activity can be divided into two stages. Stage 1 is huge masses of ignimbrites and volcanic ashes associated with interbedded andesitic to trachyandesitic lavas, erupted in early stages. After several eruptional phases, during collapse of the central domain, a caldera was formed. Stage 2 is dominant with growth of dacitic to trachyandesitic domes in the central parts of the old caldera. The Quaternary Sabalan lavas were described as high K calc-alkaline to shoshonitic by Dostal and Zebri [15] with highly differentiated REE patterns in chondrite-normalized diagrams. Didon and Germain [16] also suggested high $\mathrm{K}$ calc-alkaline and shoshonitic signatures for the Sabalan volcanic rocks.

Trachybasalts are minor in the Sabalan volcano and occur mostly as old, Miocene lava flows (Figure 2). They are distributed in the southwestern parts of the Sabalan caldera. These rocks grade upward into trachyandesitic lavas and occasionally are interbedded with pyroclastic rocks. Trachyandesites-porphyritic andesites occur mostly in the southern to western parts of the Sabalan caldera and are associated with pumiceous andesites. Trachytes are characterized by the presence of amphibole and biotite crystals. These rocks usually are vesicular. Quaternary porphyritic trachyandesites are mostly distributed in the central parts of the caldera and 


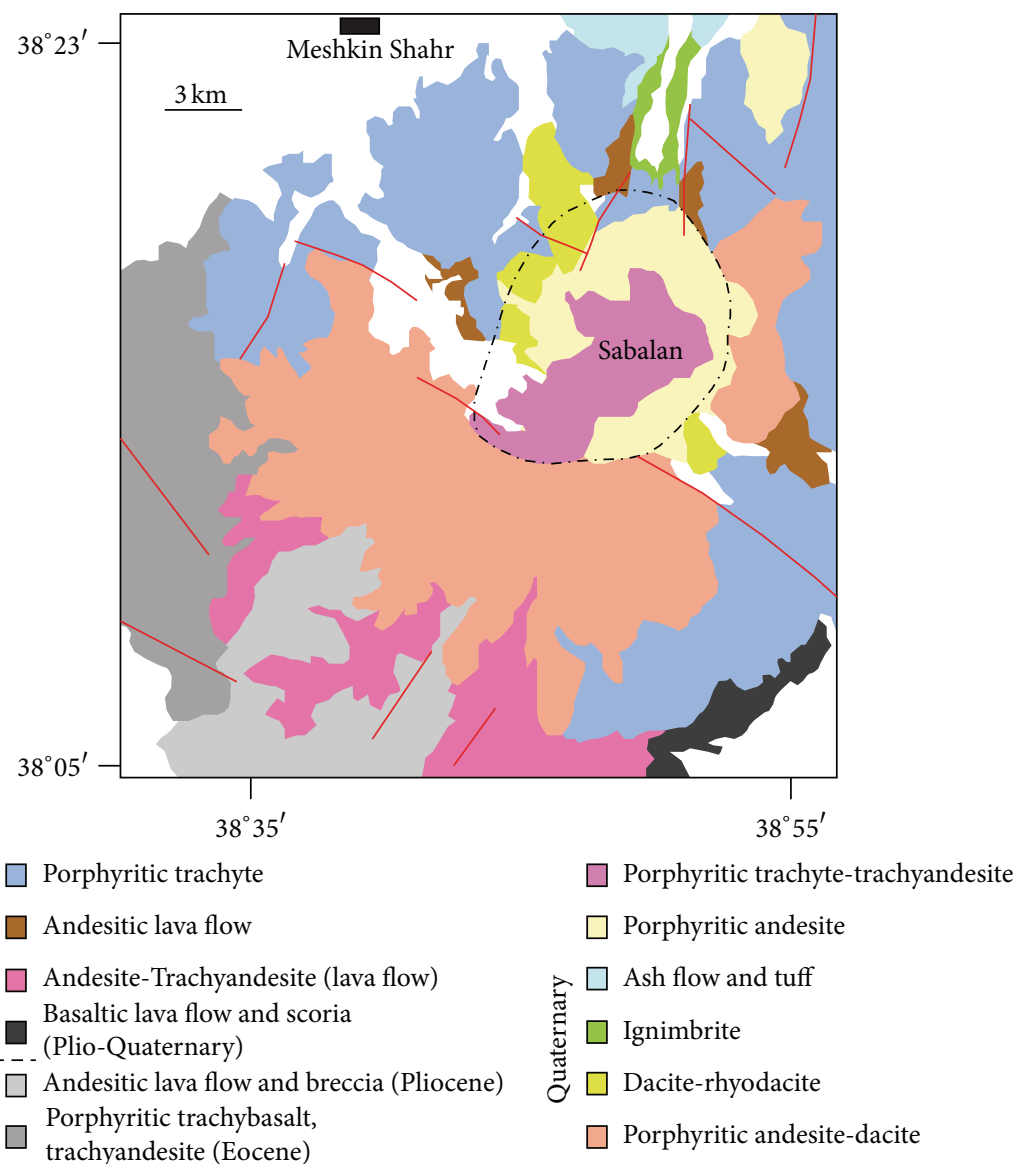

Figure 2: Simplified geological map of the Sabalan region with emphasis on the distribution of Sabalan Miocene to Quaternary and Paleogene volcanic rocks (modified after 1/100000 Geological Map of Meshkin Shahr, Geological Survey of Iran).

are the main constituents of the Quaternary Sabalan lavas (Figure 2). These lavas (trachyandesites) are intebedded with ignimberites. Dacites are minor in the Sabalan region and occur mostly as aphyric lavas associated with trachyandesites.

Ignimbrites constitute the major components of the pyroclastic eruptions and contain compressed fiammes with andesitic composition. Pumice and scoria flows are common. Lahar deposits, showing the latest stages of the volcanic activity, are predominant in the Sabalan region.

\section{Petrography of Sabalan Quaternary Volcanic Rocks}

Here, we adopted a classification based on the occurrence type of the rocks in the field, petrographical studies and mineral modes. In this way, we subdivided the rock samples into six groups.

(1) Trachyandesites. These rocks are porphyritic with large $(1-2 \mathrm{~cm}) \mathrm{K}$-feldspar phenocrysts with slight alteration into clay minerals. Biotite is another rockforming mineral and occurs as euhedral to subhedral phenocrysts. Plagioclase shows zoning and sometimes alteration into clay minerals and sericite. The groundmass indicates alteration into clay and chlorite, pseudomorph phases after glass shards. Plagioclase and K-feldspar microlites occur in the groundmass. Amphibole in trachyandesites is rare while clinopyroxene in more mafic varieties is common. These rocks show hyaloporphyritic texture.

(2) Dacitic Lavas. K-feldspar, plagioclase, biotite, and quartz are predominant phases of dacitic lavas. These minerals occur as small crystals within groundmass and/or as microphenocrysts. K-feldspar and plagioclase show slight alteration into sericite and clay minerals. Biotite displays brown paleochroism and is fresh. These rocks are mostly aphyric and most samples show holohyaline texture.

(3) Trachytes. Trachytes have more K-feldspar than plagioclase and biotite and no quartz compared to dacites. Plagioclase phenocrysts show oscillatory to patchy zoning. K-feldspar shows slight alteration into clay minerals. Plagioclase microlites and glass shards are common in the groundmass. These rocks show intersertal to hyalo-microlitic porphyritic texture.

(4) Andesites. Andesites are characterized by higher amounts of plagioclase than K-feldspar and occurrence of clinopyroxene microphenocrysts. Plagioclases show normal to oscillatory zoning. Plagioclases 
are less altered into clay than K-feldspar. Clinopyroxenes are subhedral to anhedral and occasionally show twining. Plagioclase microlites are dominant in the groundmass of the rocks.

(5) Ignimbrites. These rocks are characterized by high amount of biotite associated with K-feldspar, plagioclase, and rarely quartz with flowed glass shards (eutaxitic texture). These rocks sometimes show layering, dark layers rich in biotite, and white layers rich in feldspar, due to the gravity flow of the crystal aggregates after explosion of the volcano. Oxidized andesitic fiammes are characteristic of some ignimbrite flows.

(6) Pumice and Scoria. Andesitic scoria flows with large and abundant vesicles are common. Pumices and compressed pumices with fiammes are other rocks that accompany the pyroclastic units. Andesitic scoria flows have large amounts of glass, with rare plagioclase and clinopyroxene microphenocrysts.

\section{Geochemistry of Quaternary Lavas}

4.1. Analytical Methods. For this study we selected ten fresh samples from Quaternary Sabalan volcanic rocks for major, trace, and REE elements analyzed at ALS, Canada, using ICP-AES for major elements and ICP-MS for trace and REE elements. The uncertainty ( 1 sigma) is $\sim 2 \%$ for major elements and 5 to $10 \%$ for trace elements (depending on concentration). Mineral analyses have been performed at Paris VI University using Cameca SX 100. Accelerating voltage, beam current, and beam diameter for the analyses were $20 \mathrm{kV}, 20 \mathrm{nA}$, and $3 \mu \mathrm{m}$, respectively.

The isotopic analyses were carried out at the Laboratório de Geologia Isotópica da Universidade de Aveiro (Portugal). For Sr-Nd isotopes, samples were dissolved with $\mathrm{HF} / \mathrm{HNO}_{3}$ in Teflon Parr acid digestion bombs at $200^{\circ} \mathrm{C}$. After evaporation of the final solution, the samples were dissolved with $\mathrm{HCl}(6 \mathrm{~N})$ and dried down. The elements for analysis were purified using a conventional two-stage ion chromatography technique: (i) separation of $\mathrm{Sr}$ and REE elements in ion exchange column with AG8 $50 \mathrm{~W}$ Bio-Rad cation exchange resin; (ii) purification of $\mathrm{Nd}$ from other lanthanide elements in columns with Ln resin (ElChroM Technologies) cation exchange resin. All reagents used in sample preparation were subboiling distilled, and water was produced by a Milli-Q Element (Millipore) apparatus. Sr was loaded on a single Ta filament with $\mathrm{H}_{3} \mathrm{PO}_{4}$, whereas $\mathrm{Nd}$ was loaded on a $\mathrm{Ta}$ side filament with $\mathrm{HCl}$, in a triple filament arrangement. Both elements were determined using a Multi-Collector Thermal Ionisation Mass Spectrometer (TIMS) VG Sector 54. Data were obtained in dynamic mode with peak measurements at $1-2 \mathrm{~V}$ for ${ }^{88} \mathrm{Sr}$ and $0.8-1.5 \mathrm{~V}$ for ${ }^{144} \mathrm{Nd}$. Sr and $\mathrm{Nd}$ isotopic ratios were corrected for mass fractionation relative to ${ }^{88} \mathrm{Sr} /{ }^{86} \mathrm{Sr}=0.1194$ and ${ }^{146} \mathrm{Nd} /{ }^{144} \mathrm{Nd}$ $=0.7219$. During this study, the SRM-987 standard gave an average value of ${ }^{87} \mathrm{Sr} /{ }^{86} \mathrm{Sr}=0.710263$ (6) $(N=20$; $95 \%$ c.l. $)$ and the JNdi-1 standard yielded ${ }^{143} \mathrm{Nd} /{ }^{144} \mathrm{Nd}=0.512098$ (2)

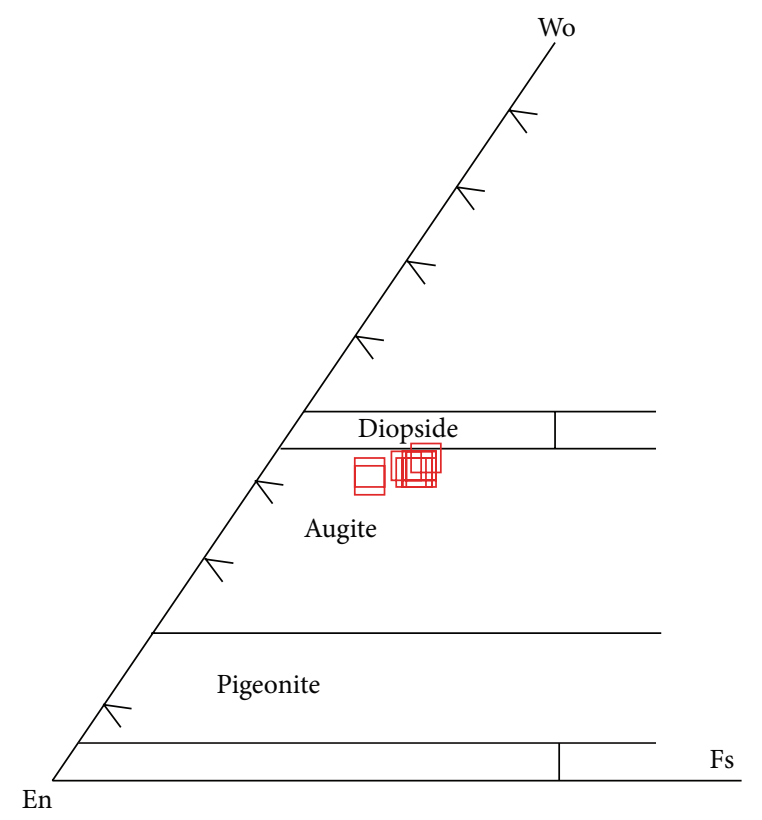

(a)

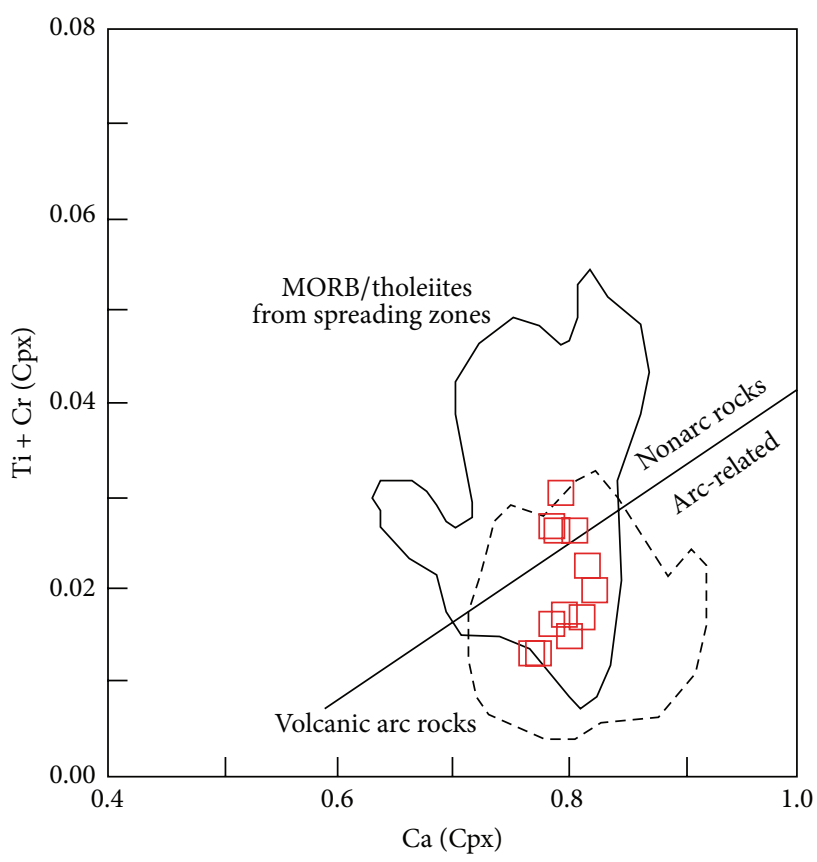

(b)

Figure 3: (a) Pyroxene composition of the Sabalan lavas in Wo-EnFs diagram. (b) Composition of pyroxenes in a plot of $\mathrm{Ti}+\mathrm{Cr}$ versus $\mathrm{Ca}$ [17], indicating their formation in an arc-related setting.

$(N=29 ; 95 \%$ c.l. $)\left({ }^{143} \mathrm{Nd} /{ }^{144} \mathrm{Nd}\right.$ data are normalized to the La Jolla Nd standard).

4.2. Mineral Chemistry. In this section, we focus on mineral chemistry of Quaternary Sabalan trachyandesites, the predominant rocks in the region. 
4.2.1. Plagioclase. Plagioclases in trachyandesites are characterized by andesine to labradorite composition (37 to 65\% An, Table 1). Their $\mathrm{K}_{2} \mathrm{O}$ content ranges between 0.3 and $1.4 \mathrm{wt} \%$ (Table 1). Their variable An content indicates spots from various bands of zoning within mineral.

4.2.2. Clinopyroxene. Clinopyroxene of trachyandesites are characterized by augitic composition (Figure 3(a)). Enstatite (En) end-member of these clinopyroxenes ranges from 43 to $48 \%$ while their ferrosillite (Fs) component is low, varying between 10 and $15 \%$ (Table 1 ). $\mathrm{TiO}_{2}$ and $\mathrm{Al}_{2} \mathrm{O}_{3}$ range between $0.28-0.95$ and 1.1-2.8 wt\%, respectively, while $\mathrm{Cr}_{2} \mathrm{O}_{3}$ and $\mathrm{Na}_{2} \mathrm{O}$ contents show lower values of $0.1-0.7$ and $0.3-$ $0.7 \mathrm{wt} \%$, respectively (Table 1 ). Their $\mathrm{Ti}$ and $\mathrm{Na}$ contents are different from those in alkaline rocks, characterized by high concentrations of these elements (titano-augite or aegirineaugite). In $\mathrm{Ti}+\mathrm{Cr}$ versus $\mathrm{Ca}$ diagram of [17], trachyandesite clinopyroxenes have a tendency to plot in volcanic arc field (Figure 3(b)).

4.2.3. Glass Shards. Glass shards in the groundmass of trachyandesites have minor traces of alteration and then were selected for EMP analysis. Their $\mathrm{SiO}_{2}$ content is variable, from 66.7 to $75.7 \mathrm{wt} \%$ (Table 1), higher than $\mathrm{SiO}_{2}$ content of whole rocks (57.6-65.4 wt\%). The $\mathrm{K}_{2} \mathrm{O}$ content of these glasses is between 3.6 and $8.1 \mathrm{wt} \%$, with low content of $\mathrm{MgO}(0.003-$ $0.07 \mathrm{wt} \%)$ and $\mathrm{TiO}_{2}(0.2-1.1 \mathrm{wt} \%)$.

4.3. Whole Rock Chemistry. The Sabalan lavas have slight alteration, characterized by low content of LOI values (1.1$1.9 \mathrm{wt} \%$, Table 2), except sample S08-7 with $3.2 \mathrm{wt} \%$ LOI. The lavas are intermediate to acidic in composition, with $\mathrm{SiO}_{2}$ content ranging from 57.6 to $65.4 \mathrm{wt} \%$ (Table 2). In total alkalis versus $\mathrm{SiO}_{2}$ diagram of [18], the Sabalan lavas (bulk rock) show tendency to plot in trachyandesitic to trachydacitic fields, similar to other shoshonitic lavas from NW Iran (Figure 4). These lavas tend to plot in trachydacite and rhyolite domains based on the composition of the glass shards of the rock groundmass. $\mathrm{K}_{2} \mathrm{O}$ and $\mathrm{TiO}_{2}$ contents of the Sabalan lavas vary between $2.5-4.8$ and $0.7-0.9 \mathrm{wt} \%$ (Table 2). In $\mathrm{K}_{2} \mathrm{O}$ against $\mathrm{SiO}_{2}$ diagram [19], the lavas show affinity to high-K calc-alkaline and shoshonitic rocks. This geochemical signature is common for other postcollisional rocks from NW Iran and NE Turkey [2] (Figure 5). In chondrite-normalized REE diagram (Figure 6), Sabalan lavas show a fractioned REE trend, with enrichment in LREEs relative to HREEs $\left(\mathrm{La}_{(\mathrm{n})} / \mathrm{Yb}_{(\mathrm{n})}=18.8-20.4\right)$. Some samples show slight depletion in $\mathrm{Eu}$, indicating plagioclase fractionation.

The Sabalan lavas show conspicuous depletion in $\mathrm{Nb}$, $\mathrm{Ta}, \mathrm{Ti}$, and $\mathrm{P}$ (e.g., $\mathrm{Nb}_{(\mathrm{n})} / \mathrm{La}_{(\mathrm{n})}=0.5-0.6$ ) with positive anomalies in most LIL elements including $\mathrm{Rb}, \mathrm{Th}, \mathrm{U}$, and $\mathrm{Pb}$ (e.g., $\mathrm{Th}_{(\mathrm{n})} / \mathrm{La}_{(\mathrm{n})}=1.4-2.9$ and $\left.\mathrm{U}_{(\mathrm{n})} / \mathrm{La}_{(\mathrm{n})}=1.5-3.2\right)$ (Figure 6). These rare earth and trace elements patterns are mostly consistent with shoshonitic signature of these rocks, indicating magmatism along an active continental margin.

4.4. Nd-Sr Isotopes. Trachyandesitic samples have uniform initial $(t=2 \mathrm{Ma}){ }^{87} \mathrm{Sr} /{ }^{86} \mathrm{Sr}$ values $(0.704-0.705)$ with $\varepsilon \mathrm{Nd}$

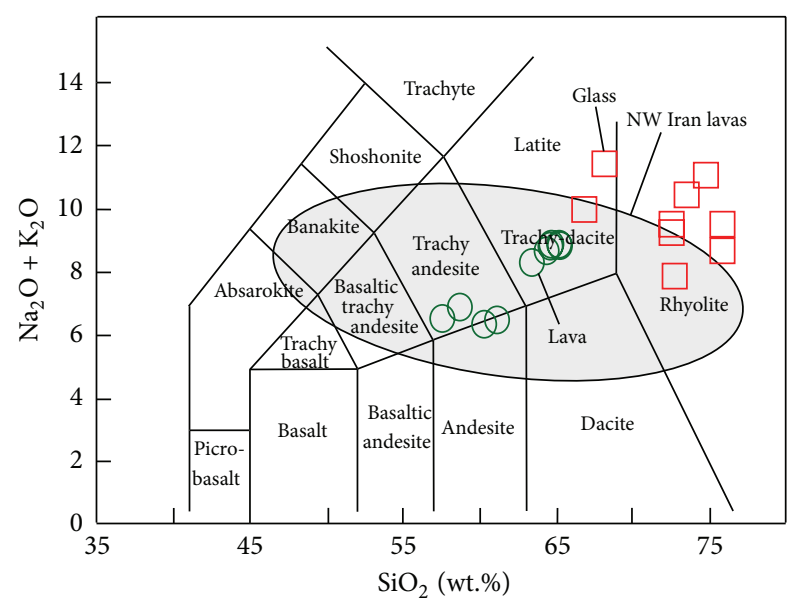

FIgURE 4: Total alkali versus $\mathrm{SiO}_{2}$ classification diagram of the Sabalan volcanic rocks [18]. Data source of NW Iran lavas is from [2].

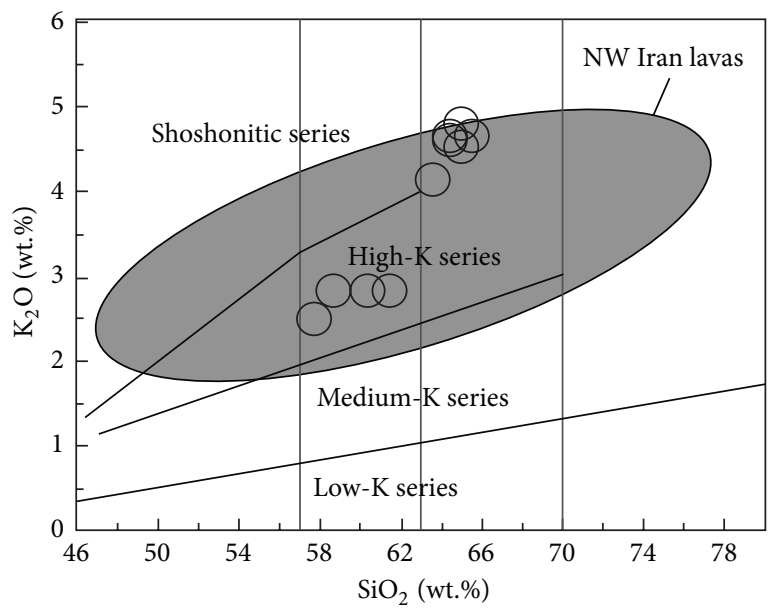

Figure 5: $\mathrm{K}_{2} \mathrm{O}$ versus $\mathrm{SiO}_{2}$ diagram [19] of Sabalan volcanic rocks. Data source of NW Iran lavas is from [2].

ranging from $\sim+1$ to +1.8 . In the initial ${ }^{87} \mathrm{Sr} /{ }^{86} \mathrm{Sr}$ versus $\varepsilon \mathrm{Nd}$ variation diagram (Figure 7), the Sabalan volcanic rocks are characterized by derivation from an enriched mantle source, and all samples show tendency to plot in a field defined by island-arc (IAB) and ocean-island (OIB) basalts. The samples mostly show affinity to plot near the Ghoshchi alkali gabbros (unpublished data), Suffi-abad I-type granites [21], and Eastern Pontides adakites [22] (Figure 7). The Sabalan shoshonitic lavas have higher $\varepsilon N d$ than Quaternary Salmas lavas (unpublished data), indicating mantle source of the Sabalan lavas slightly depleted compared to that of Salmas lavas.

\section{Discussion}

5.1. Petrogenesis. The Sabalan volcanic rocks are characterized by a fractionated REE pattern, with high LREE/HREE ratio, resembling shoshonitic rocks from active continental 
TABLE 1: Composition of clinopyroxene, plagioclase, and glass shards in the Sabalan volcanic rocks.

(a)

\begin{tabular}{|c|c|c|c|c|c|c|c|c|c|c|c|c|c|}
\hline Name & S2 & $\mathrm{S} 2$ & S5 & S5 & S5 & S9 & S9 & S9 & S9 & S9 & S4 & S4 & S4 \\
\hline Mineral & Cpx & Cpx & Cpx & Cpx & Cpx & Cpx & Cpx & Cpx & Cpx & Cpx & Cpx & Cpx & Cpx \\
\hline $\mathrm{SiO}_{2}$ & 53.16 & 52.94 & 51.33 & 52.40 & 52.56 & 50.13 & 52.32 & 51.67 & 52.81 & 50.47 & 50.64 & 52.66 & 52.16 \\
\hline $\mathrm{TiO}_{2}$ & 0.82 & 0.47 & 0.76 & 0.95 & 0.42 & 0.80 & 0.46 & 0.71 & 0.55 & 0.95 & 0.59 & 0.28 & 0.45 \\
\hline $\mathrm{Al}_{2} \mathrm{O}_{3}$ & 2.22 & 1.99 & 2.44 & 2.52 & 1.34 & 2.67 & 1.22 & 1.93 & 1.11 & 2.10 & 2.78 & 2.71 & 2.51 \\
\hline $\mathrm{Cr}_{2} \mathrm{O}_{3}$ & 0.14 & 0.00 & 0.31 & 0.00 & 0.16 & 0.00 & 0.00 & 0.02 & 0.00 & 0.00 & 0.02 & 0.72 & 0.18 \\
\hline $\mathrm{FeO}^{*}$ & 8.26 & 8.94 & 8.74 & 8.93 & 9.47 & 8.80 & 9.07 & 8.38 & 8.86 & 9.44 & 8.39 & 6.36 & 6.46 \\
\hline $\mathrm{MnO}$ & 0.37 & 0.74 & 0.57 & 0.51 & 0.59 & 0.55 & 0.36 & 0.19 & 0.53 & 0.42 & 0.36 & 0.31 & 0.21 \\
\hline $\mathrm{MgO}$ & 15.07 & 15.01 & 14.67 & 14.29 & 14.84 & 14.87 & 14.78 & 15.19 & 15.39 & 15.35 & 15.37 & 16.60 & 16.81 \\
\hline $\mathrm{CaO}$ & 20.05 & 19.37 & 19.80 & 19.93 & 19.70 & 20.10 & 19.16 & 20.51 & 20.12 & 20.06 & 20.24 & 19.60 & 20.19 \\
\hline $\mathrm{Na}_{2} \mathrm{O}$ & 0.46 & 0.46 & 0.59 & 0.45 & 0.49 & 0.37 & 0.34 & 0.46 & 0.42 & 0.68 & 0.32 & 0.38 & 0.40 \\
\hline $\mathrm{K}_{2} \mathrm{O}$ & 0.00 & 0.01 & 0.00 & 0.00 & 0.00 & 0.02 & 0.01 & 0.05 & 0.03 & 0.05 & 0.05 & 0.01 & 0.07 \\
\hline $\mathrm{NiO}$ & 0.07 & 0.19 & 0.00 & 0.03 & 0.00 & 0.00 & 0.00 & 0.01 & 0.18 & 0.03 & 0.06 & 0.08 & 0.00 \\
\hline $\mathrm{BaO}$ & 0.28 & 0.00 & 0.00 & 0.00 & 0.00 & 0.00 & 0.21 & 0.00 & 0.00 & 0.00 & 0.00 & 0.43 & 0.14 \\
\hline $\mathrm{F}$ & 0.00 & 0.00 & 0.00 & 0.25 & 0.08 & 0.00 & 0.12 & 0.00 & 0.16 & 0.12 & 0.00 & 0.04 & 0.00 \\
\hline $\mathrm{Cl}$ & 0.00 & 0.00 & 0.02 & 0.05 & 0.09 & 0.00 & 0.00 & 0.00 & 0.00 & 0.05 & 0.03 & 0.00 & 0.00 \\
\hline Total & 100.89 & 100.10 & 99.23 & 100.32 & 99.74 & 98.31 & 98.05 & 99.11 & 100.14 & 99.70 & 98.83 & 100.18 & 99.57 \\
\hline $\mathrm{Mg}^{\#}$ & 0.76 & 0.75 & 0.75 & 0.74 & 0.74 & 0.75 & 0.74 & 0.76 & 0.76 & 0.74 & 0.77 & 0.82 & 0.82 \\
\hline $\mathrm{Cr}^{\#}$ & 0.04 & 0.00 & 0.08 & 0.00 & 0.07 & 0.00 & 0.00 & 0.01 & 0.00 & 0.00 & 0.00 & 0.15 & 0.04 \\
\hline En & 0.44 & 0.44 & 0.43 & 0.43 & 0.43 & 0.43 & 0.44 & 0.44 & 0.44 & 0.44 & 0.44 & 0.48 & 0.48 \\
\hline Fs & 0.14 & 0.15 & 0.15 & 0.15 & 0.15 & 0.14 & 0.15 & 0.14 & 0.14 & 0.15 & 0.14 & 0.10 & 0.10 \\
\hline Wo & 0.42 & 0.41 & 0.42 & 0.43 & 0.41 & 0.42 & 0.41 & 0.43 & 0.42 & 0.41 & 0.42 & 0.41 & 0.42 \\
\hline $\mathrm{Si}$ & 1.95 & 1.96 & 1.93 & 1.94 & 1.97 & 1.90 & 1.98 & 1.94 & 1.96 & 1.90 & 1.91 & 1.94 & 1.93 \\
\hline $\mathrm{Ti}$ & 0.02 & 0.01 & 0.02 & 0.03 & 0.01 & 0.02 & 0.01 & 0.02 & 0.02 & 0.03 & 0.02 & 0.01 & 0.01 \\
\hline $\mathrm{Al}$ & 0.10 & 0.09 & 0.11 & 0.11 & 0.06 & 0.12 & 0.05 & 0.09 & 0.05 & 0.09 & 0.12 & 0.12 & 0.11 \\
\hline $\mathrm{Cr}$ & 0.00 & 0.00 & 0.01 & 0.00 & 0.00 & 0.00 & 0.00 & 0.00 & 0.00 & 0.00 & 0.00 & 0.02 & 0.01 \\
\hline $\mathrm{Fe}^{*}$ & 0.25 & 0.28 & 0.27 & 0.28 & 0.30 & 0.28 & 0.29 & 0.26 & 0.28 & 0.30 & 0.26 & 0.20 & 0.20 \\
\hline $\mathrm{Mn}$ & 0.01 & 0.02 & 0.02 & 0.02 & 0.02 & 0.02 & 0.01 & 0.01 & 0.02 & 0.01 & 0.01 & 0.01 & 0.01 \\
\hline $\mathrm{Mg}$ & 0.83 & 0.83 & 0.82 & 0.79 & 0.83 & 0.84 & 0.83 & 0.85 & 0.85 & 0.86 & 0.86 & 0.91 & 0.93 \\
\hline $\mathrm{Ca}$ & 0.79 & 0.77 & 0.80 & 0.79 & 0.79 & 0.82 & 0.78 & 0.82 & 0.80 & 0.81 & 0.82 & 0.77 & 0.80 \\
\hline $\mathrm{Na}$ & 0.03 & 0.03 & 0.04 & 0.03 & 0.04 & 0.03 & 0.03 & 0.03 & 0.03 & 0.05 & 0.02 & 0.03 & 0.03 \\
\hline $\mathrm{K}$ & 0.00 & 0.00 & 0.00 & 0.00 & 0.00 & 0.00 & 0.00 & 0.00 & 0.00 & 0.00 & 0.00 & 0.00 & 0.00 \\
\hline $\mathrm{Ni}$ & 0.00 & 0.01 & 0.00 & 0.00 & 0.00 & 0.00 & 0.00 & 0.00 & 0.01 & 0.00 & 0.00 & 0.00 & 0.00 \\
\hline Total & 3.99 & 4.00 & 4.02 & 3.99 & 4.01 & 4.03 & 3.99 & 4.02 & 4.01 & 4.05 & 4.03 & 4.00 & 4.02 \\
\hline
\end{tabular}

(b)

\begin{tabular}{|c|c|c|c|c|c|c|c|c|c|c|c|c|}
\hline Name & $\mathrm{S} 2$ & $\mathrm{~S} 2$ & $\mathrm{~S} 2$ & S9 & S9 & S9 & S9 & S5 & S5 & $\mathrm{S} 4$ & $\mathrm{~S} 4$ & S4 \\
\hline Mineral & Plag & Plag & Plag & Plag & Plag & Plag & Plag & Plag & Plag & Plag & Plag & Plag \\
\hline $\mathrm{SiO}_{2}$ & 56.984 & 57.374 & 57.797 & 58.691 & 58.503 & 58.330 & 55.219 & 51.093 & 50.666 & 52.338 & 52.592 & 55.365 \\
\hline $\mathrm{TiO}_{2}$ & 0.162 & 0.067 & 0.080 & 0.080 & 0.117 & 0.052 & 0.073 & 0.073 & 0.022 & 0.058 & 0.000 & 0.022 \\
\hline $\mathrm{Al}_{2} \mathrm{O}_{3}$ & 26.210 & 26.159 & 25.617 & 25.602 & 24.411 & 23.408 & 26.422 & 29.910 & 29.617 & 28.542 & 28.959 & 27.208 \\
\hline $\mathrm{Cr}_{2} \mathrm{O}_{3}$ & 0.000 & 0.063 & 0.000 & 0.032 & 0.000 & 0.000 & 0.032 & 0.000 & 0.114 & 0.032 & 0.000 & 0.292 \\
\hline $\mathrm{FeO}$ & 0.542 & 0.593 & 0.683 & 0.450 & 0.536 & 0.400 & 0.596 & 0.533 & 0.938 & 0.691 & 0.517 & 1.065 \\
\hline $\mathrm{MnO}$ & 0.061 & 0.000 & 0.000 & 0.087 & 0.000 & 0.000 & 0.000 & 0.000 & 0.000 & 0.000 & 0.000 & 0.000 \\
\hline $\mathrm{MgO}$ & 0.028 & 0.017 & 0.013 & 0.007 & 0.023 & 0.023 & 0.040 & 0.030 & 0.101 & 0.061 & 0.055 & 0.088 \\
\hline $\mathrm{CaO}$ & 8.802 & 8.795 & 7.656 & 7.085 & 7.094 & 7.526 & 9.544 & 12.947 & 12.451 & 12.060 & 12.005 & 9.777 \\
\hline $\mathrm{Na}_{2} \mathrm{O}$ & 5.583 & 5.826 & 6.191 & 6.558 & 6.683 & 6.862 & 5.769 & 4.245 & 3.722 & 4.808 & 4.873 & 5.364 \\
\hline $\mathrm{K}_{2} \mathrm{O}$ & 0.942 & 1.006 & 1.203 & 1.083 & 1.341 & 1.443 & 0.918 & 0.261 & 0.255 & 0.425 & 0.313 & 0.597 \\
\hline $\mathrm{NiO}$ & 0.000 & 0.041 & 0.041 & 0.000 & 0.055 & 0.000 & 0.000 & 0.136 & 0.000 & 0.055 & 0.000 & 0.150 \\
\hline $\mathrm{BaO}$ & 0.000 & 0.000 & 0.143 & 0.215 & 0.358 & 0.000 & 0.071 & 0.071 & 0.215 & 0.071 & 0.144 & 0.071 \\
\hline
\end{tabular}


(b) Continued.

\begin{tabular}{lcccccccccccc}
\hline Name & S2 & S2 & S2 & S9 & S9 & S9 & S9 & S5 & S5 & S4 & S4 & S4 \\
Mineral & Plag & Plag & Plag & Plag & Plag & Plag & Plag & Plag & Plag & Plag & Plag & Plag \\
\hline $\mathrm{F}$ & 0.000 & 0.000 & 0.000 & 0.205 & 0.041 & 0.123 & 0.124 & 0.168 & 0.000 & 0.000 & 0.000 & 0.083 \\
$\mathrm{Cl}$ & 0.000 & 0.016 & 0.005 & 0.000 & 0.026 & 0.032 & 0.016 & 0.000 & 0.068 & 0.000 & 0.011 & 0.032 \\
\hline Total & 99.314 & 99.955 & 99.430 & 100.095 & 99.189 & 98.199 & 98.824 & 99.466 & 98.169 & 99.141 & 99.469 & 100.115 \\
\hline An\% & 46.560 & 45.482 & 40.596 & 37.384 & 36.972 & 37.737 & 47.760 & 62.762 & 64.896 & 58.091 & 57.653 & 50.181 \\
\hline $\mathrm{Si}$ & 2.582 & 2.587 & 2.618 & 2.639 & 2.662 & 2.680 & 2.535 & 2.350 & 2.358 & 2.409 & 2.409 & 2.511 \\
$\mathrm{Ti}$ & 0.006 & 0.002 & 0.003 & 0.003 & 0.004 & 0.002 & 0.003 & 0.003 & 0.001 & 0.002 & 0.000 & 0.001 \\
$\mathrm{Al}$ & 1.400 & 1.390 & 1.368 & 1.357 & 1.309 & 1.267 & 1.430 & 1.621 & 1.624 & 1.548 & 1.563 & 1.454 \\
$\mathrm{Cr}$ & 0.000 & 0.002 & 0.000 & 0.001 & 0.000 & 0.000 & 0.001 & 0.000 & 0.004 & 0.001 & 0.000 & 0.010 \\
$\mathrm{Fe}{ }^{*}$ & 0.021 & 0.022 & 0.026 & 0.017 & 0.020 & 0.015 & 0.023 & 0.021 & 0.037 & 0.027 & 0.020 & 0.040 \\
$\mathrm{Mn}$ & 0.002 & 0.000 & 0.000 & 0.003 & 0.000 & 0.000 & 0.000 & 0.000 & 0.000 & 0.000 & 0.000 & 0.000 \\
$\mathrm{Mg}$ & 0.002 & 0.001 & 0.001 & 0.000 & 0.002 & 0.002 & 0.003 & 0.002 & 0.007 & 0.004 & 0.004 & 0.006 \\
$\mathrm{Ca}$ & 0.427 & 0.425 & 0.372 & 0.341 & 0.346 & 0.370 & 0.469 & 0.638 & 0.621 & 0.595 & 0.589 & 0.475 \\
$\mathrm{Na}$ & 0.491 & 0.509 & 0.544 & 0.572 & 0.590 & 0.611 & 0.514 & 0.379 & 0.336 & 0.429 & 0.433 & 0.472 \\
$\mathrm{~K}$ & 0.054 & 0.058 & 0.070 & 0.062 & 0.078 & 0.085 & 0.054 & 0.015 & 0.015 & 0.025 & 0.018 & 0.035 \\
$\mathrm{Ni}$ & 0.000 & 0.001 & 0.001 & 0.000 & 0.002 & 0.000 & 0.000 & 0.005 & 0.000 & 0.002 & 0.000 & 0.005 \\
\hline Total & 4.985 & 4.998 & 5.002 & 4.996 & 5.013 & 5.032 & 5.031 & 5.034 & 5.003 & 5.042 & 5.035 & 5.009 \\
\hline
\end{tabular}

(c)

\begin{tabular}{lccccccccc}
\hline Name & S2 & S2 & S5 & S5 & S9 & S9 & S9 & S4 \\
Mineral & Glass & Glass & Glass & Glass & Glass & Glass & Glass & Glass \\
\hline $\mathrm{SiO}_{2}$ & 75.32 & 75.748 & 73.41 & 74.528 & 68.305 & 72.28 & 66.705 & 72.307 & 72.793 \\
$\mathrm{TiO}_{2}$ & 0.829 & 0.827 & 0.807 & 0.756 & 0.212 & 0.952 & 0.33 & 1.113 \\
$\mathrm{Al}_{2} \mathrm{O}_{3}$ & 12.863 & 12.567 & 14.018 & 14.063 & 17.432 & 13.495 & 18.55 & 14.152 & 1.146 \\
$\mathrm{Cr}_{2} \mathrm{O}_{3}$ & 0.064 & 0.048 & 0.032 & 0.032 & 0.032 & 0.114 & 0 & 0 & 0.08 \\
$\mathrm{FeO}$ & 0.773 & 1.853 & 0.773 & 0.732 & 0.578 & 1.4 & 0.908 & 1.424 & 2.7 \\
$\mathrm{MnO}$ & 0 & 0 & 0.009 & 0.043 & 0.094 & 0 & 0 & 0 & 0.035 \\
$\mathrm{MgO}$ & 0.041 & 0.041 & 0.003 & 0.025 & 0.036 & 0.051 & 0.046 & 0.051 & 0.065 \\
$\mathrm{CaO}$ & 0.315 & 0.259 & 0.186 & 0.259 & 1.087 & 0.316 & 2.163 & 0.327 & 0.227 \\
$\mathrm{Na}$ & 2.59 & 2.205 & 2.536 & 2.99 & 5.346 & 3.317 & 6.42 & 2.062 & 2.092 \\
$\mathrm{~K} 2 \mathrm{O}$ & 6.125 & 7.171 & 7.93 & 8.169 & 6.145 & 5.993 & 3.645 & 7.377 & 5.853 \\
$\mathrm{NiO}$ & 0 & 0.027 & 0 & 0 & 0.055 & 0.041 & 0 & 0.055 \\
$\mathrm{BaO}$ & 0 & 0 & 0.071 & 0 & 0.644 & 0 & 0 & 0 \\
$\mathrm{~F}$ & 0.21 & 0 & 0.042 & 0 & 0.041 & 0.376 & 0.285 & 0 & 0 \\
$\mathrm{Cl}$ & 0.203 & 0.117 & 0.096 & 0.074 & 0.058 & 0.165 & 0.085 & 0.159 & 0.042 \\
\hline $\mathrm{Total}$ & 99.343 & 100.863 & 99.727 & 101.671 & 100.065 & 98.5 & 99.139 & 99.028 & 99.063 \\
\hline
\end{tabular}

$\mathrm{Mg}^{\#}: \mathrm{Mg}$ number, Feo $^{*}$ : total iron.

margins and/or postcollisional magmatism after closure of oceanic basins. Depletion in high field strength elements and enrichment in large lithophile elements are conspicuous. Such shoshonitic volcanism is characteristic of the TurkishIranian high plateau $[2,24]$. The glass shards in the groundmass of these rocks are characterized by more fractionated nature, with higher $\mathrm{SiO}_{2}$ and $\mathrm{K}_{2} \mathrm{O}$ contents, indicating quenching products of more-evolved melt after fractionation of mafic minerals. Trachyandesite clinopyroxenes have a tendency to plot at volcanic arc rocks field in $\mathrm{Ti}+\mathrm{Cr}$ versus Ca diagram, indicating an arc-related environment for the formation of Sabalan volcanic rocks. These characteristics show that these clinopyroxenes are crystallized from an arcrelated calc-alkaline magma. The Sabalan lavas are similar to Goshchi alkali gabbros both of them are characterized by derivation from an isotopically enriched mantle source (OIB mantle source). They are clearly different from crustal contaminated, postcollisional Islamic peninsula shoshonites and ultrapotassic rocks. When compared to other Iranian postcollisional Plio-Quaternary lavas, like the Salmas basalts, the Sabalan rocks show higher $\varepsilon N d$ values, clearly indicating a slight depleted mantle source for the Sabalan lavas and/or contamination of the Salmas lavas with crustal materials during their ascent to the surface. The interaction with crustal rocks and/or derivation from a subduction-contaminated source is also clear from $\mathrm{Th} / \mathrm{Yb}$ versus $\mathrm{Ta} / \mathrm{Yb}$ diagram [25] for the Sabalan lavas (Figure 8). In this diagram, the Sabalan lavas show higher $\mathrm{Th} / \mathrm{Yb}$ ratio, far from within plate mantle trend, 
TABLE 2: Whole rock and Sr-Nd isotope composition of the Sabalan volcanic rocks.

\begin{tabular}{|c|c|c|c|c|c|c|c|c|c|c|}
\hline Name & S08-3 & S08-1 & S08-2 & S08-4 & S08-5 & S08-6 & S08-7 & S08-8 & S08-9 & S08-10 \\
\hline $\mathrm{SiO}_{2}$ & 61.2 & 58.8 & 65.4 & 60.4 & 64.9 & 57.6 & 63.5 & 64.4 & 64.6 & 65.2 \\
\hline $\mathrm{Al}_{2} \mathrm{O}_{3}$ & 16.8 & 16.8 & 15 & 16.5 & 15.2 & 16.5 & 14.6 & 14.9 & 14.95 & 15 \\
\hline FeOt & 5.09 & 6.06 & 3.69 & 5.08 & 3.97 & 6.08 & 3.62 & 3.73 & 3.82 & 3.75 \\
\hline $\mathrm{CaO}$ & 5.45 & 6.17 & 2.62 & 5.37 & 2.64 & 5.8 & 2.61 & 2.69 & 2.73 & 2.65 \\
\hline $\mathrm{MgO}$ & 2.4 & 2.46 & 1.09 & 2.41 & 1.08 & 2.99 & 1.08 & 1.1 & 1.1 & 1.06 \\
\hline $\mathrm{Na}_{2} \mathrm{O}$ & 3.57 & 4.01 & 4.11 & 3.52 & 4.25 & 3.97 & 4.08 & 4.06 & 4.03 & 4.02 \\
\hline $\mathrm{K}_{2} \mathrm{O}$ & 2.86 & 2.83 & 4.68 & 2.81 & 4.6 & 2.49 & 4.17 & 4.58 & 4.69 & 4.78 \\
\hline $\mathrm{TiO}_{2}$ & 0.7 & 0.89 & 0.7 & 0.7 & 0.71 & 0.88 & 0.68 & 0.71 & 0.71 & 0.7 \\
\hline $\mathrm{MnO}$ & 0.1 & 0.1 & 0.09 & 0.1 & 0.09 & 0.11 & 0.09 & 0.09 & 0.09 & 0.08 \\
\hline $\mathrm{P}_{2} \mathrm{O}_{5}$ & 0.27 & 0.42 & 0.25 & 0.26 & 0.25 & 0.41 & 0.24 & 0.25 & 0.28 & 0.25 \\
\hline LOI & 1.54 & 1.68 & 1.32 & 1.49 & 1.88 & 1.09 & 3.24 & 1.36 & 1.59 & 1.45 \\
\hline Total & 100 & 100.5 & 99.2 & 98.8 & 99.8 & 98.1 & 98.1 & 98 & 98.7 & 99.1 \\
\hline $\mathrm{Ba}$ & 844 & 1500 & 829 & 809 & 1140 & 784 & 1015 & 748 & 744 & 775 \\
\hline $\mathrm{Ce}$ & 78.5 & 98.1 & 127.5 & 78.3 & 127.5 & 90 & 124.5 & 125.5 & 123.5 & 125 \\
\hline $\mathrm{Cr}$ & 60 & 90 & 450 & 40 & 140 & 90 & 80 & 30 & 230 & 20 \\
\hline Cs & 2.07 & 1.63 & 5.07 & 2.01 & 4.98 & 1.5 & 4.92 & 4.81 & 4.78 & 4.89 \\
\hline Dy & 3.15 & 3.84 & 4.06 & 3.18 & 4.2 & 3.55 & 4.04 & 3.99 & 4.06 & 4.06 \\
\hline $\mathrm{Er}$ & 1.88 & 2.27 & 2.48 & 1.87 & 2.6 & 2.01 & 2.47 & 2.5 & 2.45 & 2.56 \\
\hline $\mathrm{Eu}$ & 1.29 & 1.65 & 1.36 & 1.31 & 1.4 & 1.53 & 1.33 & 1.37 & 1.39 & 1.35 \\
\hline Gd & 4.62 & 5.78 & 6.27 & 4.54 & 6.43 & 5.29 & 6.14 & 6.13 & 6.15 & 6.18 \\
\hline $\mathrm{Hf}$ & 4.9 & 5.7 & 8.7 & 4.8 & 9 & 5.1 & 8.5 & 8.6 & 8.5 & 8.7 \\
\hline Ho & 0.64 & 0.77 & 0.82 & 0.63 & 0.85 & 0.7 & 0.84 & 0.81 & 0.82 & 0.83 \\
\hline $\mathrm{La}$ & 46.6 & 57.5 & 73.4 & 46.3 & 73.5 & 52 & 71.9 & 72 & 70.4 & 72 \\
\hline $\mathrm{Lu}$ & 0.27 & 0.32 & 0.41 & 0.27 & 0.42 & 0.27 & 0.39 & 0.39 & 0.39 & 0.4 \\
\hline $\mathrm{Nb}$ & 20.9 & 28.3 & 48.8 & 21 & 49.3 & 25.1 & 47.1 & 46.9 & 46.4 & 47.5 \\
\hline $\mathrm{Nd}$ & 27.6 & 35.4 & 41.4 & 27.4 & 41.5 & 32.3 & 41 & 40.7 & 40.7 & 40.4 \\
\hline $\mathrm{Ni}$ & 28 & 32 & 8 & 28 & 9 & 36 & 6 & 7 & 8 & 7 \\
\hline $\mathrm{Pb}$ & 13 & 16 & 21 & 14 & 23 & 13 & 20 & 24 & 19 & 23 \\
\hline $\mathrm{Pr}$ & 7.9 & 10.05 & 12.45 & 7.92 & 12.5 & 9.1 & 12.4 & 12.4 & 12.25 & 12.3 \\
\hline $\mathrm{Rb}$ & 72 & 71.5 & 180.5 & 72.4 & 180 & 64.5 & 173.5 & 176.5 & 176 & 179 \\
\hline $\mathrm{Sm}$ & 4.79 & 5.88 & 6.61 & 4.66 & 6.62 & 5.46 & 6.52 & 6.58 & 6.46 & 6.44 \\
\hline $\mathrm{Sr}$ & 626 & 837 & 401 & 622 & 410 & 751 & 448 & 415 & 421 & 415 \\
\hline $\mathrm{Ta}$ & 1.4 & 1.8 & 3.4 & 1.4 & 3.6 & 1.6 & 3.3 & 3.4 & 3.3 & 3.4 \\
\hline $\mathrm{Tb}$ & 0.61 & 0.75 & 0.79 & 0.59 & 0.83 & 0.69 & 0.78 & 0.77 & 0.77 & 0.78 \\
\hline Th & 9.28 & 10.1 & 26.3 & 9.2 & 26.7 & 9.08 & 25.7 & 25.5 & 25.1 & 25.8 \\
\hline $\mathrm{Tm}$ & 0.26 & 0.31 & 0.38 & 0.26 & 0.38 & 0.28 & 0.36 & 0.36 & 0.36 & 0.37 \\
\hline $\mathrm{U}$ & 2.75 & 2.67 & 7.11 & 2.72 & 7.11 & 2.37 & 6.87 & 6.89 & 6.81 & 6.98 \\
\hline $\mathrm{V}$ & 100 & 121 & 59 & 99 & 58 & 118 & 58 & 58 & 60 & 57 \\
\hline $\mathrm{Y}$ & 17.7 & 21.2 & 23.7 & 17.9 & 23.9 & 19.2 & 23.5 & 23.4 & 23.3 & 23.6 \\
\hline $\mathrm{Yb}$ & 1.78 & 2.02 & 2.61 & 1.77 & 2.7 & 1.85 & 2.58 & 2.55 & 2.52 & 2.56 \\
\hline $\mathrm{Zn}$ & 72 & 89 & 102 & 98 & 81 & 89 & 72 & 95 & 68 & 92 \\
\hline $\mathrm{Zr}$ & 210 & 253 & 368 & 211 & 376 & 230 & 356 & 356 & 354 & 357 \\
\hline${ }^{87} \mathrm{Sr} /{ }^{86} \mathrm{Sr}$ & & & & & & & 0.70511 & & 0.70452 & 0.70443 \\
\hline${ }^{143} \mathrm{Nd} /{ }^{144} \mathrm{Nd}$ & & & & & & & 0.51273 & & 0.51269 & 0.51271 \\
\hline$\varepsilon \mathrm{Nd}$ & & & & & & & 1.8 & & 1.04 & 1.4 \\
\hline
\end{tabular}

resembling those lavas that erupted above an active continental margin. Higher Th content could have also resulted from interaction of ascending lavas with crustal rocks, via assimilation-fractional crystallization (AFC) processes. The
Sabalan lavas seem to show contamination with high Th (and high ${ }^{87} \mathrm{Sr} /{ }^{86} \mathrm{Sr}$ ) crustal rocks during their ascent to the surface beneath the volcano. This could be inferred from rare, partially dissolved crustal xenoliths in the Sabalan lavas, 

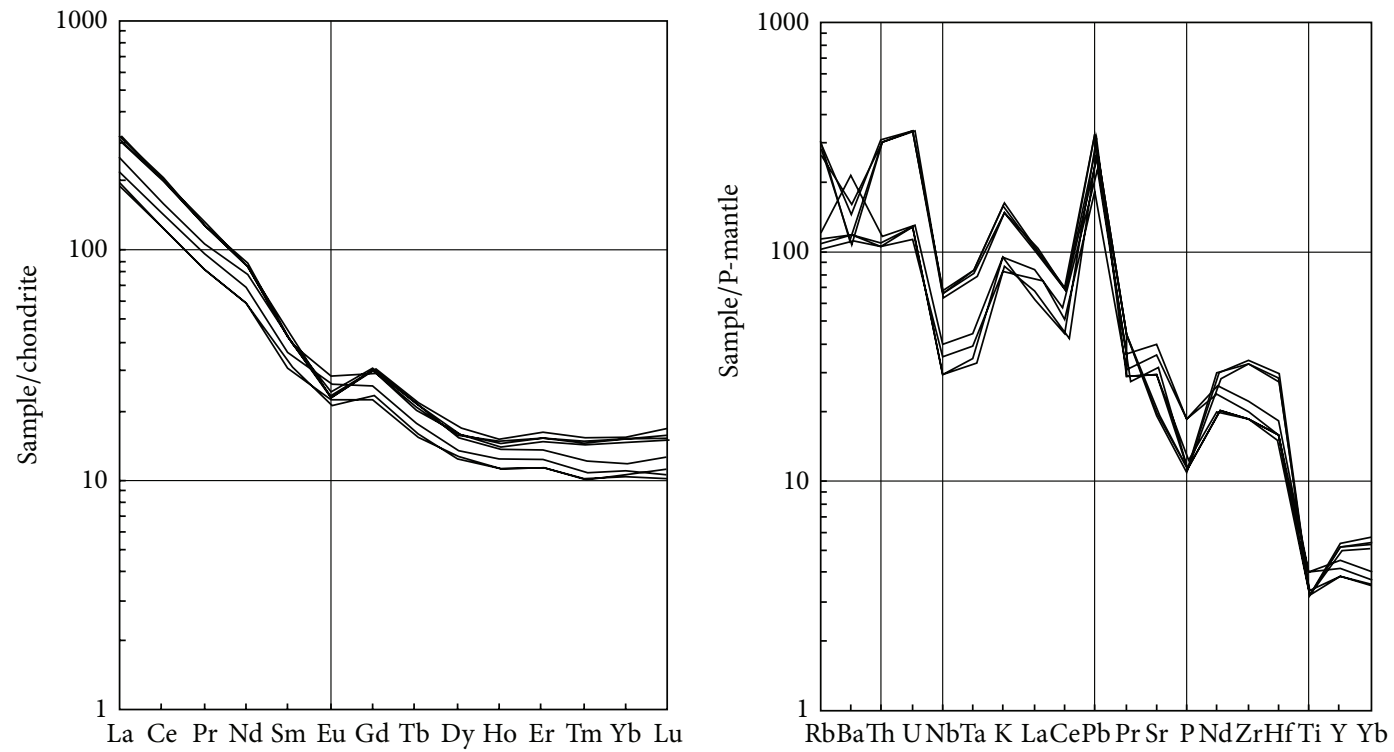

FIGURE 6: Chondrite-normalized REE and mantle-normalized trace elements patterns of the Sabalan volcanic rocks. Normalization data are from [20].
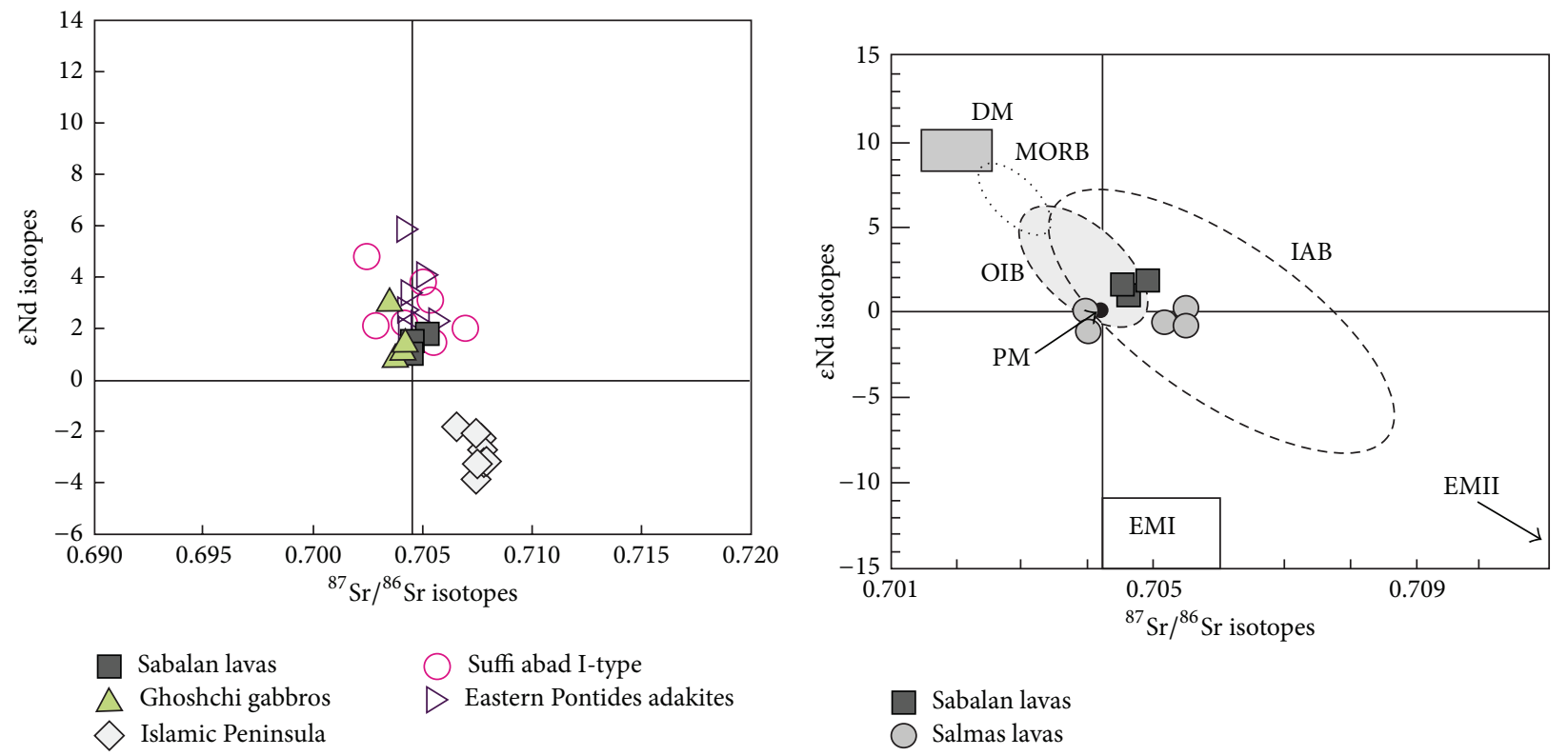

Figure 7: $\varepsilon N d$ versus ${ }^{87} \mathrm{Sr} /{ }^{86} \mathrm{Sr}$ diagrams for Sabalan lavas. Data from other Turkish-Iranian postcollisional intrusive and extrusive rocks are shown for comparison; right-hand diagram is modified after [23].

which can be evidence for crust contamination. In order to evaluate the mantle-melting regime of the Sabalan lavas, we used the $\mathrm{Sm} / \mathrm{Yb}$ versus La/Sm diagram [26]. The Sabalan lavas are relatively similar to Salmas Quaternary basalts, characterized by low degree of partial melting $(<0.1 \%)$ of a mantle source containing clinopyroxene-garnet lherzolites (Figure 9). Because the Sabalan lavas are characterized by higher $\mathrm{La} / \mathrm{Sm}$ ratio, it seems that their mantle source contains more modal garnet than model source in Figure 9 and/or contains other minor phases including phlogopite and/or apatite.
5.2. Geodynamic Setting. Plio-Quaternary (to Quaternary) volcanism in the Peri-Arabian region (in Turkish-Iranian high plateau) postdates the subduction of Neotethyan lithosphere since $\sim 13 \mathrm{Ma}$ [2]. These volcanic rocks become more alkaline with time and towards the south $[2,4,5,27]$. However, most of volcanic rocks, like the Salmas and/or Maku Quaternary lavas, still show a SSZ geochemical signature including enrichment in LILE and/or depletion in HFSE, despite the lack of subduction system at that time. Geochemical and geodynamic evidence also shows a subduction setting for the genesis of Sabalan lavas: fractionated REEs 


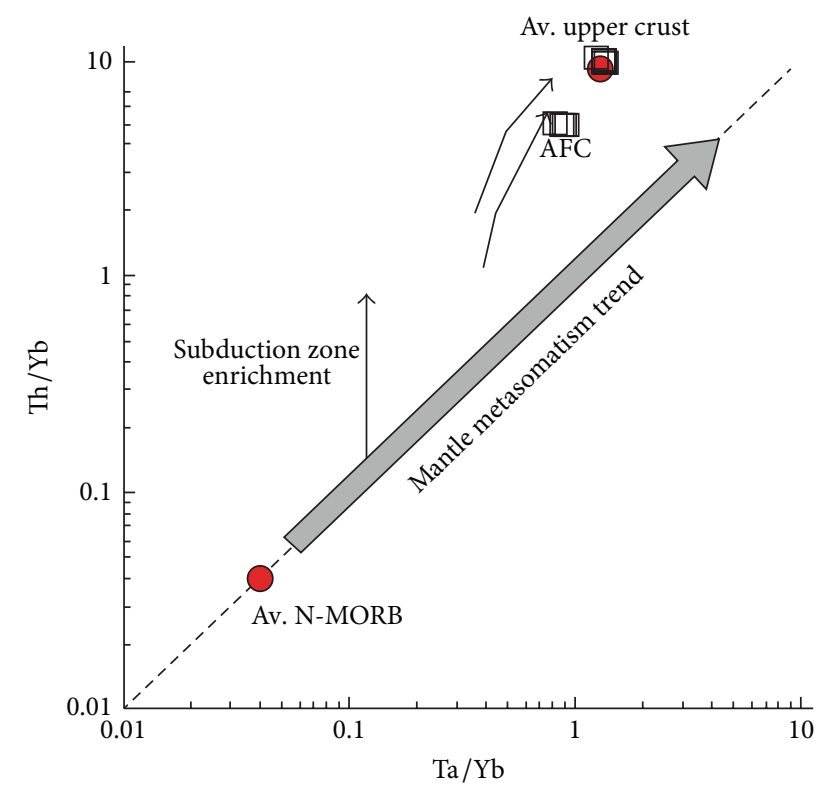

Figure 8: Th/Yb versus $\mathrm{Ta} / \mathrm{Yb}$ diagram [25] for the Sabalan Quaternary lavas.

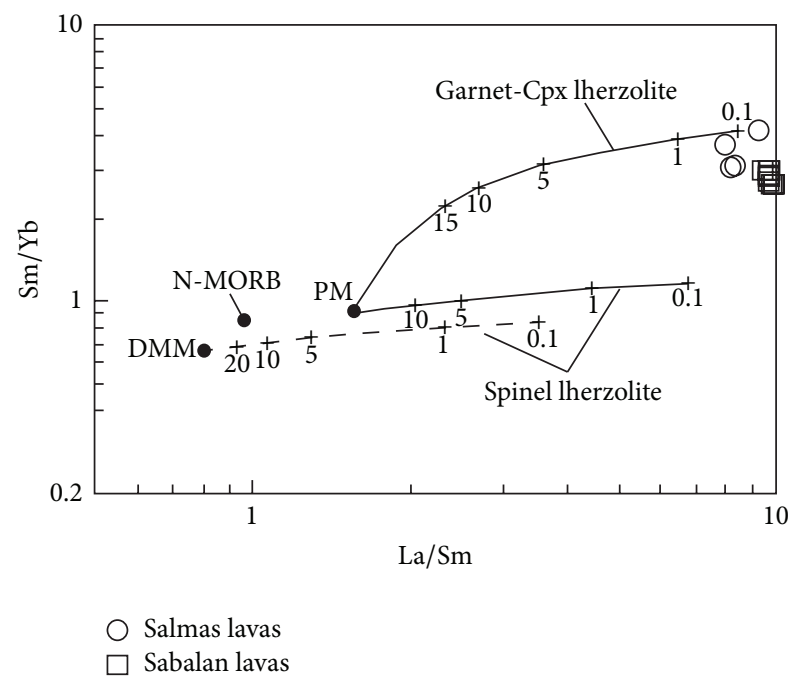

FIgURE 9: Sm/Yb versus La/Sm diagram for the Sabalan volcanic rocks with melting curves obtained using the nonmodal batch melting modelling, modified after [26].

patterns (high LREEs/HREEs ratio), enrichment in Th, Ba, $\mathrm{Rb}, \mathrm{U}, \mathrm{Pb}$, and depletion in $\mathrm{Nb}$, Ta, and Ti. Such geochemical characteristics are common in subduction-related environments [25]. These observations and isotopic characteristics of the lavas suggest that their magmas were probably derived from partial melting of a subduction-metasomatized continental lithospheric mantle in the garnet/spinel lherzolite field [2]. Widespread volcanism across the Turkish-Iranian plateau throughout the late Cenozoic until Plio-Quaternary is strictly ascribed to postcollisional mantle upwelling during slab steeping and breakoff beneath a subduction-accretion complex $[4,28]$.

\section{Conclusions}

The Sabalan Quaternary volcanism includes a sequence of trachyandesites, latites, trachytes with huge bodies of ignimbrites, and pyroclastic rocks. Plagioclase with andesine to labradorite and clinopyroxene with augitic composition are predominant phases in these volcanic rocks. Lavas show shoshonitic geochemical signature with a fractioned REEs trend, enrichment in LREEs relative to HREEs, and depletion in HFSEs. The Sabalan volcanic rocks are isotopically characterized by derivation from an enriched mantle source (with/without crustal influence). All samples show tendency to plot in a field defined by island-arc (IAB) and oceanisland (OIB) basalts, in terms of their $\varepsilon N d$ value and ${ }^{87} \mathrm{Sr} /{ }^{86} \mathrm{Sr}$ ratio. The lavas are characterized by higher $\mathrm{Th} / \mathrm{Yb}$ ratio, resembling those lavas that erupted above an active continental margin with geochemical evolution via assimilationfractional crystallization (AFC) process. The geochemical and isotopic signatures of the Sabalan lavas suggest that their melts have been issued via low degree partial melting of a subduction-metasomatized continental lithospheric mantle during Neotethyan slab steeping and break-off beneath the Turkish-Iranian high plateau.

\section{Conflict of Interests}

The author declare that there is no conflict of interests. Hereby I confirm that I have not any type of conflict of interests in this paper.

\section{Acknowledgments}

This study was supported by a research Grant from University of Mohagheghe Ardabili.

\section{References}

[1] Y. Dilek and D. L. Whitney, "Cenozoic crustal evolution in central Anatolia: extension, magmatism and landscape development," in Proceedings of the 3rd International Conference on the Geology of the Eastern Mediterranean, pp. 183-192, 2000.

[2] Y. Dilek, N. Imamverdiyev, and Ş. Altunkaynak, "Geochemistry and tectonics of Cenozoic volcanism in the Lesser Caucasus (Azerbaijan) and the peri-Arabian region: collision-induced mantle dynamics and its magmatic fingerprint," International Geology Review, vol. 52, no. 4-6, pp. 536-578, 2010.

[3] J. A. Pearce, J. F. Bender, S. E. De Long et al., "Genesis of collision volcanism in Eastern Anatolia, Turkey," Journal of Volcanology and Geothermal Research, vol. 44, no. 1-2, pp. 189-229, 1990.

[4] M. Keskin, "Magma generation by slab steepening and breakoff beneath a subduction-accretion complex: an alternative model for collision-related volcanism in Eastern Anatolia, Turkey," Geophysical Research Letters, vol. 30, no. 24, pp. 1-9, 2003.

[5] M. Kheirkhah, M. B. Allen, and M. Emami, "Quaternary syn-collision magmatism from the Iran/Turkey borderlands," Journal of Volcanology and Geothermal Research, vol. 182, no. 1-2, pp. 1-12, 2009.

[6] Y. Dilek and Ş. Altunkaynak, "Geochemical and temporal evolution of Cenozoic magmatism in Western Turkey: mantle response to collision, slab break-off, and lithospheric tearing 
in an orogenic belt," Geological Society of London Special Publication, vol. 311, pp. 213-233, 2009.

[7] R. Riou, C. Dupuy, and J. Dostal, "Geochemistry of coexisting alkaline and calc-alkaline volcanic rocks from northern Azerbaijan (N.W. Iran)," Journal of Volcanology and Geothermal Research, vol. 11, no. 2-4, pp. 253-275, 1981.

[8] D. Dhont and J. Chorowicz, "Review of the neotectonics of the Eastern Turkish-Armenian Plateau by geomorphic analysis of digital elevation model imagery," International Journal of Earth Sciences, vol. 95, no. 1, pp. 34-49, 2006.

[9] Y. Özdemir, Ö. Karaoǧlu, A. Ü. Tolluoǧlu, and N. Güleç, "Volcanostratigraphy and petrogenesis of the Nemrut stratovolcano (East Anatolian High Plateau): the most recent post-collisional volcanism in Turkey," Chemical Geology, vol. 226, no. 3-4, pp. 189-211, 2006.

[10] H. E. Çubukçu, I. Ulusoy, E. Aydar et al., "Mt. Nemrut volcano (Eastern Turkey): temporal petrological evolution," Journal of Volcanology and Geothermal Research, vol. 209-210, pp. 33-60, 2012.

[11] F. Innocenti, R. Mazzuoli, G. Pasquarè, F. Radicati Di Brozolo, and L. Villari, "Tertiary and quaternary volcanism of the Erzurumkars area (Eastern Turkey): geochronological data and geodynamic evolution," Journal of Volcanology and Geothermal Research, vol. 13, no. 3-4, pp. 223-240, 1982.

[12] P. Comin-Chiaramonti, S. Meriani, R. Mosca, and S. Sinigoi, "On the occurrence of analcime in the northeastern Azerbaijan volcanics (northwestern Iran)," Lithos, vol. 12, no. 3, pp. 187-198, 1979.

[13] M. Aghazadeh, A. Castro, Z. Badrzadeh, and K. Vogt, "Postcollisional polycyclic plutonism from the Zagros hinterland: the Shaivar Dagh plutonic complex, Alborz belt, Iran," Geological Magazine, vol. 148, no. 5-6, pp. 980-1008, 2011.

[14] A. Alberti, P. Comin-Chiaramonti, G. Di Battistini, R. Fioriti, and S. Sinigei, "Crystal fractionation in the eastern Azerbaijan (Iran) lower tertiary shoshonitic suite," Neues Jahrbuch für Mineralogie, Monatshefte, vol. 1, pp. 35-48, 1981.

[15] J. Dostal and M. Zerbi, "Geochemistry of the Savalan volcano (northwestern Iran)," Chemical Geology, vol. 22, no. C, pp. 3142, 1978.

[16] J. Didon and Y. M. Germain, Le Sabalan, Volcan PlioQuaternaire de l Azerbaidjan oriental (Iran): Etude geologique et petrographique de le difice et de son environment regional [Ph.D. thesis], Docteur du 3 eme cycle. Université de Grenoble, Grenoble, France, 1976.

[17] J. Leterrier, R. Maury, P. Thonon, D. Girard, and M. Marchal, "Clinopyroxene composition as a method of identification of the magmatic affinities of paleo-volcanic series," Earth and Planetary Science Letters, vol. 59, no. 1, pp. 139-154, 1982.

[18] M. J. L. Bas, R. W. L. Maitre, A. Streckeisen, and B. Zanettin, "A chemical classification of volcanic rocks based on the total alkali-silica diagram," Journal of Petrology, vol. 27, no. 3, pp. 745750, 1986.

[19] A. Peccerillo and S. R. Taylor, "Geochemistry of eocene calcalkaline volcanic rocks from the Kastamonu area, Northern Turkey," Contributions to Mineralogy and Petrology, vol. 58, no. 1, pp. 63-81, 1976.

[20] W. F. McDonough and S.-S. Sun, "The composition of the Earth," Chemical Geology, vol. 120, no. 3-4, pp. 223-253, 1995.

[21] H. Azizi, Y. Asahara, B. Mehrabi, and S. L. Chung, "Geochronological and geochemical constraints on the petrogenesis of highK granite from the Suffi abad area, Sanandaj-Sirjan Zone, NW
Iran," Chemie der Erde-Geochemistry, vol. 71, no. 4, pp. 363376, 2011.

[22] O. Karsli, M. Ketenci, I. Uysal et al., "Adakite-like granitoid porphyries in the Eastern Pontides, NE Turkey: potential parental melts and geodynamic implications," Lithos, vol. 127, no. 1-2, pp. 354-372, 2011.

[23] C. Zhang, C. Ma, and F. Holtz, "Origin of high-Mg adakitic magmatic enclaves from the Meichuan pluton, southern Dabie orogen (central China): implications for delamination of the lower continental crust and melt-mantle interaction," Lithos, vol. 119, no. 3-4, pp. 467-484, 2010.

[24] A. Jahangiri, "Post-collisional Miocene adakitic volcanism in NW Iran: geochemical and geodynamic implications," Journal of Asian Earth Sciences, vol. 30, no. 3-4, pp. 433-447, 2007.

[25] J. A. Pearce, "Trace element characteristics of lavas from destructive plate boundaries," in Andesites, R. S. Thorpe, Ed., pp. 525-548, Wiley, New York, NY, USA, 1982.

[26] R. Bezard, R. Hébert, C. Wang, J. Dostal, J. Dai, and H. Zhong, "Petrology and geochemistry of the Xiugugabu ophiolitic massif, western Yarlung Zangbo suture zone, Tibet," Lithos, vol. 125, no. 1-2, pp. 347-367, 2011.

[27] M. Keskin, J. A. Pearce, P. D. Kempton, and P. Greenwood, "Magma-crust interactions and magma plumbing in a postcollisional setting: geochemical evidence from the Erzurum-Kars volcanic plateau, eastern Turkey," Geological Society of America Special Paper, no. 409, pp. 475-505, 2006.

[28] A. M. C. Şengör, S. Özeren, T. Genç, and E. Zor, "East Anatolian High Plateau as a mantle-supported, north-south shortened domal structure," Geophysical Research Letters, vol. 30, no. 24, pp. 1-8, 2003. 

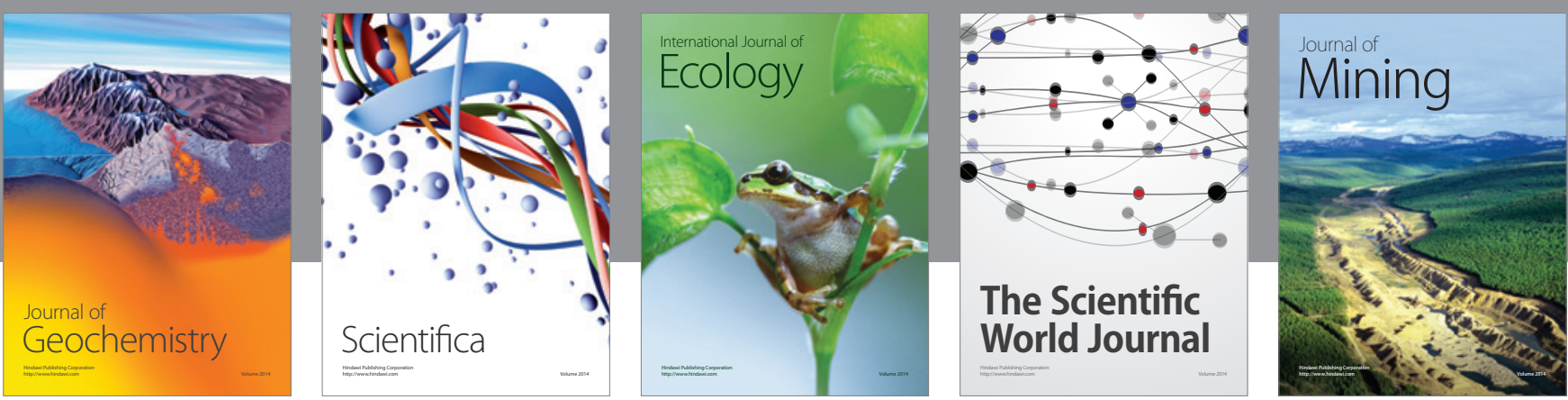

The Scientific World Journal
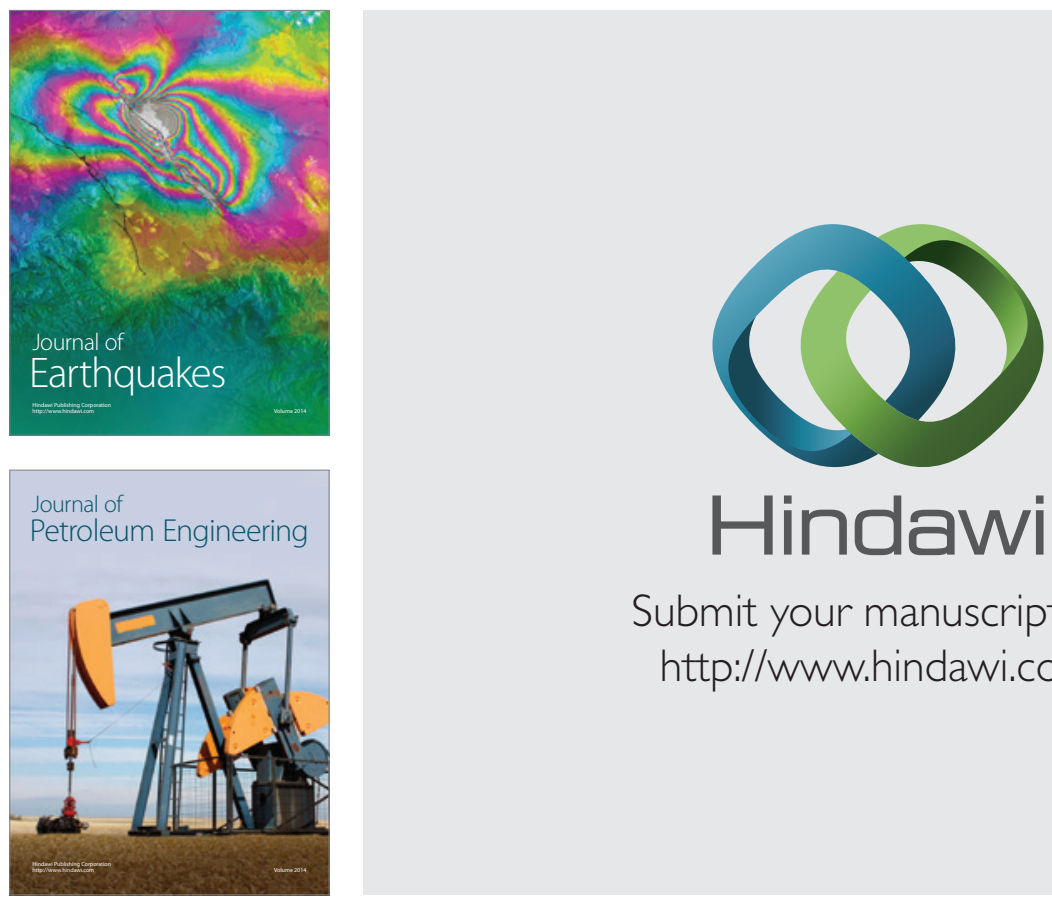

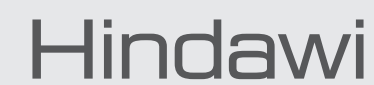

Submit your manuscripts at

http://www.hindawi.com
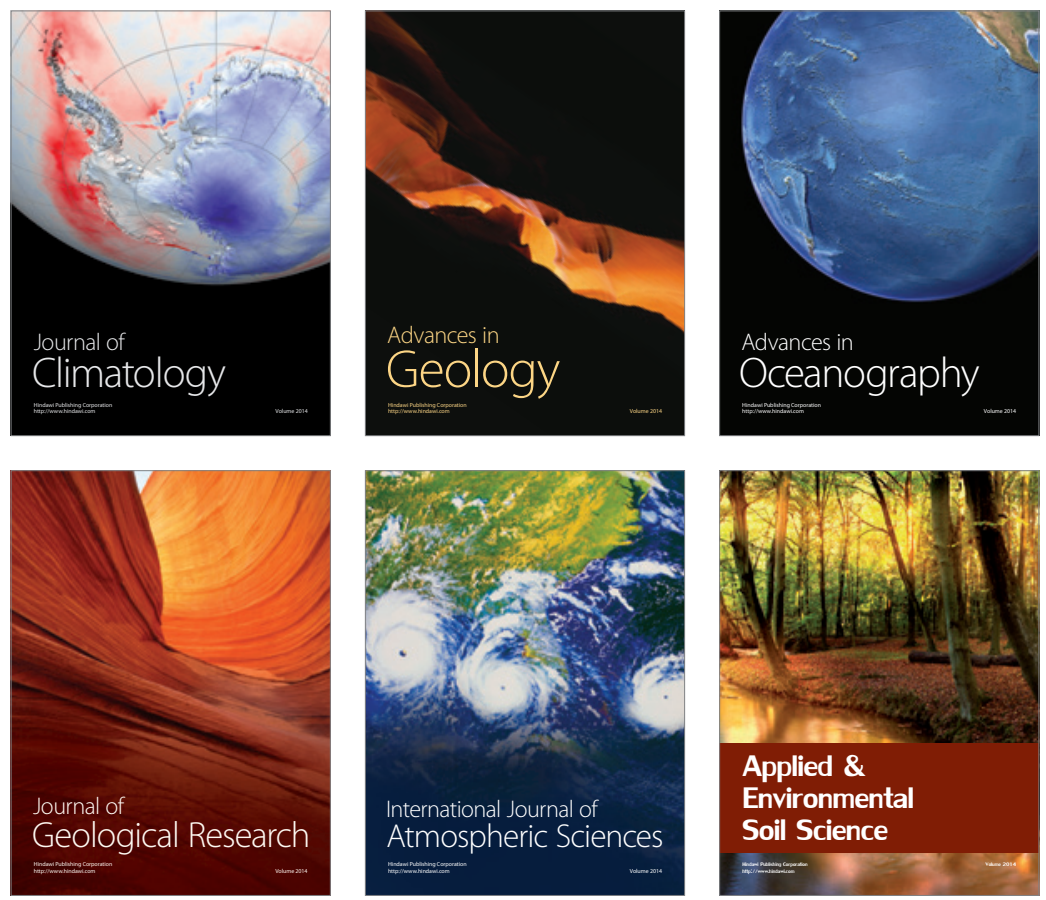
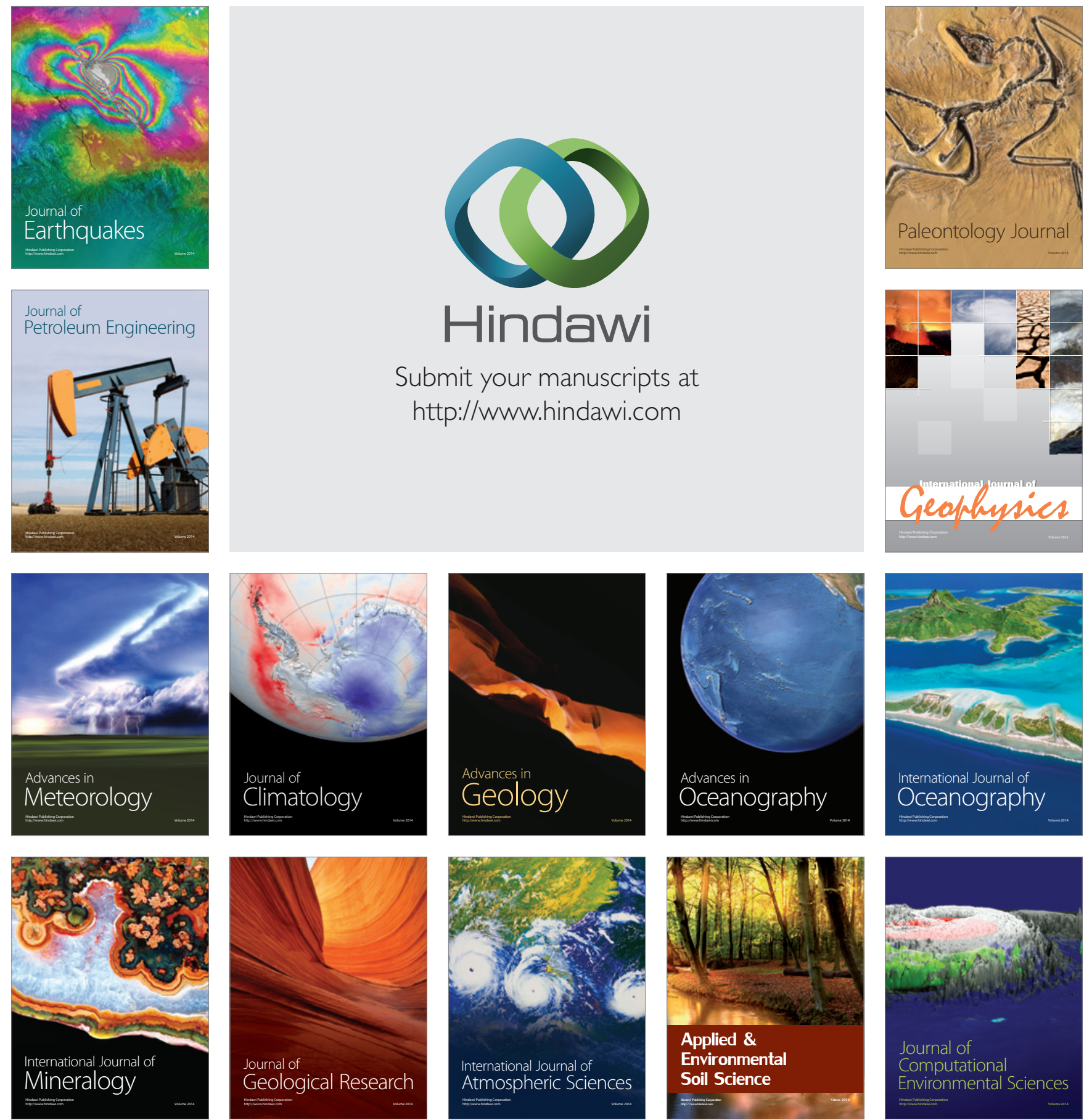